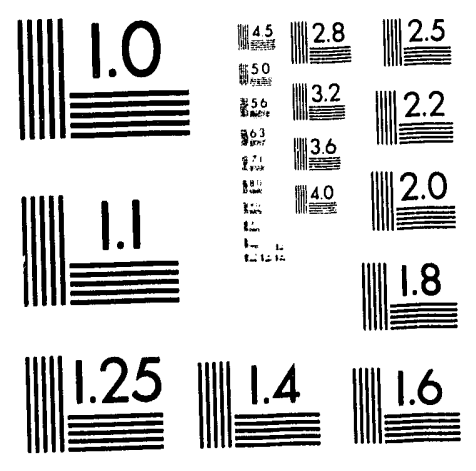



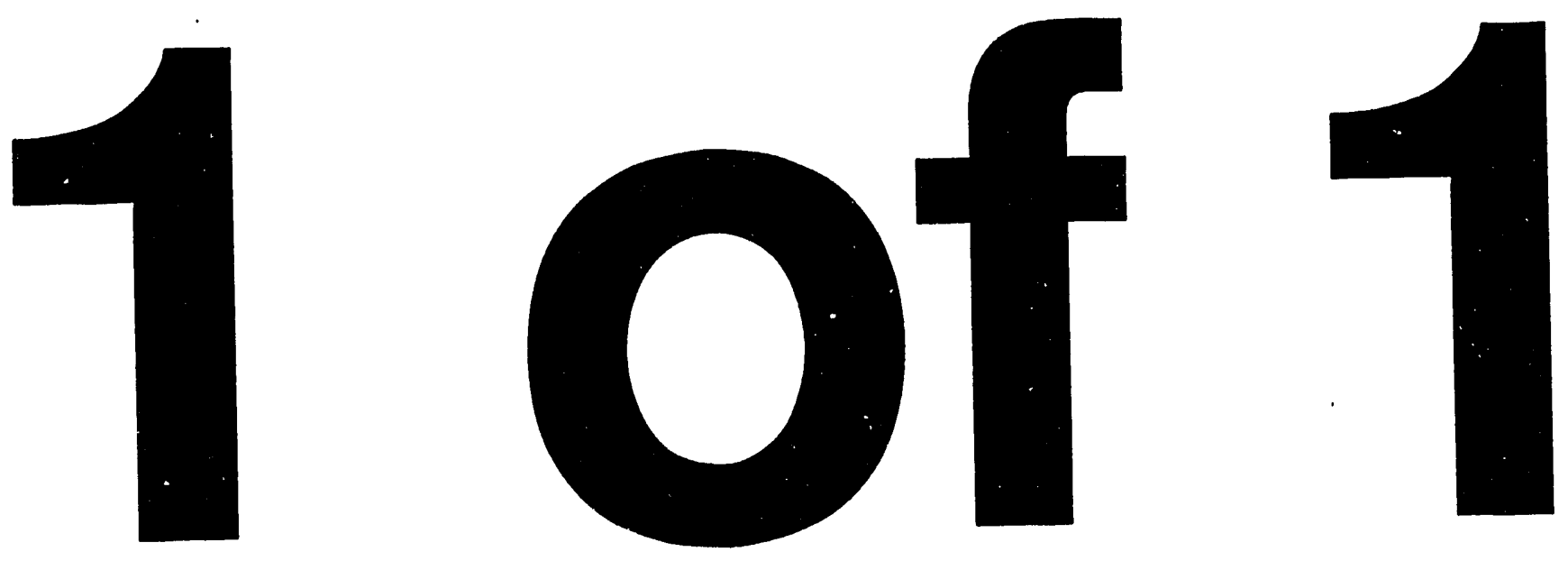


$$
D O E / P C / 90046 \ldots T /
$$

\section{HETEROGENEOUS CATALYTIC PROCESS FOR ALCOHOL FUELS FROM SYNGAS}

DOE Contract No. DE-AC22-91PC90046

Pittsburgh Energy Technology Canter

First

Quarterly Technical Progress Report

January - March, 1992

Union Carbide Chemicals and Plastics Co. Inc. South Charleston Technical Center South Charleston, West Virginia 25303 


\section{DISCLAIMER}

This Report was prepared as an account of work sponsored by the United States Government. Neither the United States nor the United States Department of Energy, nor any of their employees, makes any warranty, express or implied. or assumes any legal liability or responsibility for the accuracy, completeness, or usefulness of any information, apparatus, product, or process disclosed, or represents that its use would not infringe privately owned rights. Reference herein to any specific commercial product, process, or service by trade name, mark, manufacturer, or otherwise does not necessarily constitute or imply its endorsement, recommendation, or favoring by the United States Government or any agency thereof. The views and opinions of authors expressed herein do not necessarily state or reflect those of the United States Government or any agency thereof. 


\section{Table of Contents}

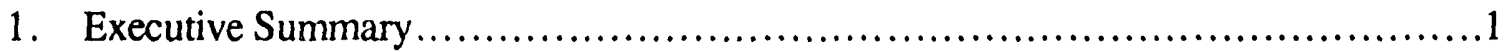

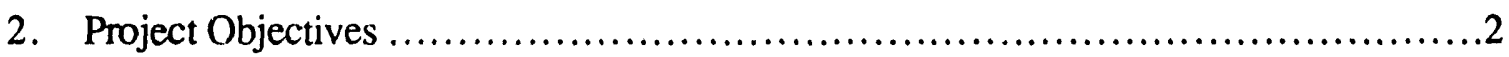

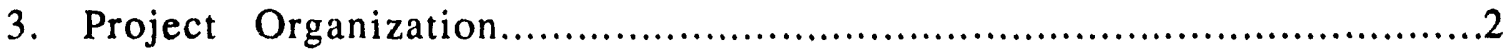

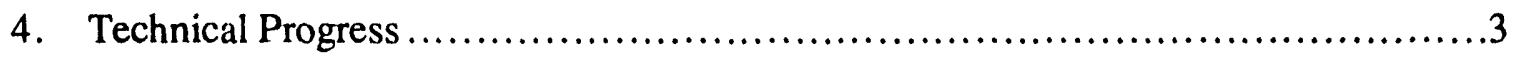

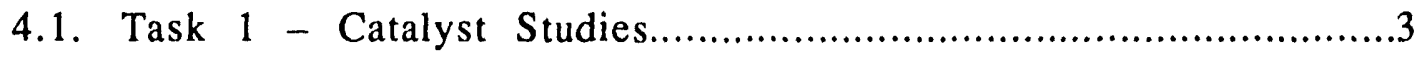

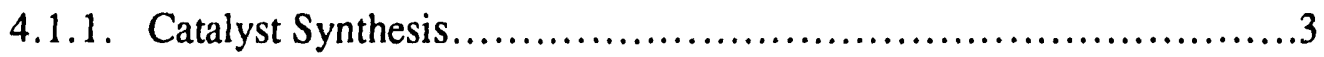

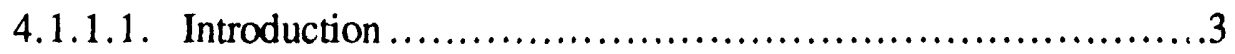

4.1.1.2. Catalyst Synthesis Results .................................

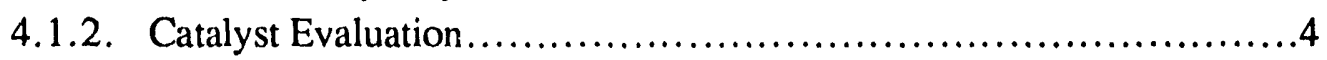

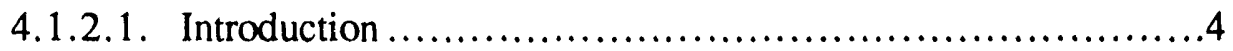

4.1.2.2. Microreactor System for Screening Catalysts..................4

4.1.2.3. Product Analysis ........................................8

4.1.2.4. Gas Chromatographic Analyses ............................... 8

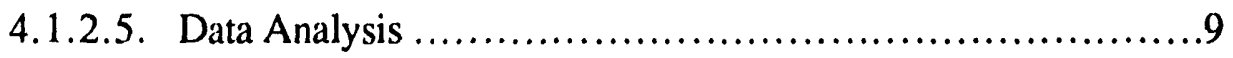

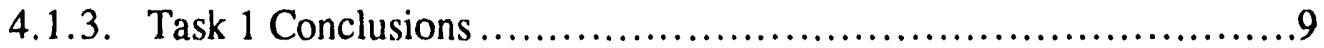

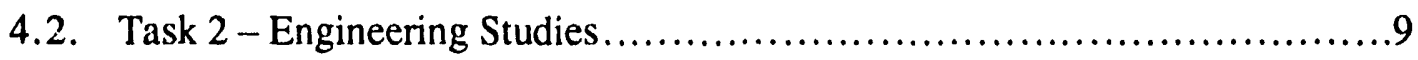

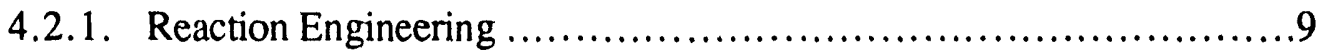

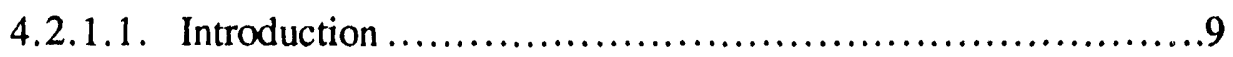

4.2.1.2. Reaction Engineering Facilities .......................... 10

4.2.2. Mathematical Process Development ................................11

4.2.2.1. Introduction ........................................... 11

4.2.2.2. Software Programs to be Used ............................. 11

4.2.3. Task 2 Conclusions ................................................ 11 


\section{Executive Summary}

The principal objectives of this project are to discover and evaluate novel heterogeneous catalysts, for conversion of syngas to oxygenates having use as fuel enhancers, to explore novel reactor and process concepts applicable in this process, and to develop the best total process for converting syngas to liquid fuels.

The project is being pursued as two concurrent tasks. Task 1 involves catalyst research and development and is being largely conducted by catalyst chemists and analytical specialists. Task 2 is largely an engineering activity, and includes process conceptualization and economics and bench-scale process evaluation of systems developed in Task 1.

Work this quarter has concentrated largely on literature work, revamping laboratory and reactor systems, and planning specific experiments to pursue the objectives of the program.

A systematic search of the literature has been initiated to ensure that our literature base on conversion of syngas to higher alcohols is current. This activity will be continued throughout the project.

Variables in catalyst formulation and preparation will be examined by a fractional factorial designed experiment. The variables are based upon previous work at UCC on a catalyst system that gave a product rich in isobutanol. The ranges of some of the fixed and free variables are being identified by a preliminary $3 \times 3$ designed set. The precipitation $\mathrm{pH}$ and its effects on surface area, porosity, and thermal characteristics of the resulting precipitate will also be investigated. Potentiometric titrations during coprecipitation are being used to aid in improving this catalyst preparation procedure.

A microreactor system has been prepared for use in screening heterogeneous catalysts in this project. It will allow the independent control of three fixed bed reactor tubes except for reaction temperature. Appropriate temperature, pressure, and flow ranges are attainable with this system.

Two reaction engineering facilities are available for this project: a catalyst preparation facility and a catalyst testing lab. In the catalyst preparation lab, catalyst powders will be converted into larger scale pellets or extrudates for further testing. In the catalyst testing lab, we will develop kinetics and test candidate catalysts in one or more of four reaction systems: (1) a microreactor system for evaluating solid catalysts in gas phase, (2) a Berty reactor for obtaining detailed kinetics from solid catalysts in gas phase, (3) a slurry reactor for evaluating powdered catalysts in a commercially feasible gas-solid-liquid system, and (4) a SpectraTech FTIR reactor for analyzing products and intermediates in situ. We may also be able to use another facility containing a multiphase reaction system to evaluate the performance of candidate catalysts in trickle bed or other multiphase reactors.

Task 2 process synthesis studies will seek to determine the most economical process flowsheet. This will involve process conceptualization and determination of the optimum operating conditions. The task can be broken into synthesis and simulation. Two software 
programs, Mayflower and Advent, are available for process synthesis. Aspen Plus software will be used to simulate a process flowsheet and determine a heat and material balance. It can simulate a variety of unit operations and provides sizing information needed to determine equipment cost. The program can also determine the sensitivity of operating variables on the process.

\section{Project Objectives}

- To discover, study, and evaluate novel heterogeneous catalytic systems for the production of oxygenated fuel enhancers from synthesis gas. In particular, novel heterogeneous catalysts will be studied and optimized for the production of: (a) $C_{1}-C_{5}$ alcohols using conventional methanol synthesis conditions, and (b) methanol and isobutanol mixtures which may be used for the downstream synthesis of MTBE or related oxygenates.

- To explore, analytically and on the bench scale, novel reactor and process concepts for use in converting syngas to liquid fuel products.

- To develop on the bench scale the best combination of chemistry, catalyst, reactor, and total process configuration to achieve the minimum product cost for the conversion of syngas to liquid products.

\section{Project Organization}

This project has been divided into two tasks.

Task 1 is concerned with catalyst identification, preparation, performance evaluation, and characterization. This work is being largely conducted by catalyst chemists and analytical specialists. Chemical studies to support the engineering effort in Task 2 are included in this task, but fundamental aspects of the catalytic chemistry are emphasized in this effort.

Task 2 includes process conceptualization and economics, and bench-scale process evaluation of systems developed in Task 1 . This is largely an engineering activity.

These tasks are being carried out concurrently throughout the project. 


\section{Technical Progress}

\subsection{Task 1 - Catalyst Studies}

\subsubsection{Catalyst Synthesis}

\subsubsection{Introduction}

Synthesis gas can be converted into mixtures of methanol and higher alcohols by use of alkali-modified methanol synthesis catalysts known for many years. Interest in higher alcohol synthesis from syngas has stemmed from the desire to use the alcohol mixtures as high-octane blending stock for gasoline. Currently refining modifications and the use of oxygenated petrochemicals such as methyl tert-butyl ether (MTBE) have been implemented as alternatives. The production of a mixture of methanol and isobutanol is of interest due to its possible use as a feedstock in the production of other oxygenates such as MTBE and related ethers.

Previous work at UCC on a methanol-isobutanol catalyst used consecutive Hyper-GrecoLatin Squares to identify and bracket levels of important variables in catalyst formulation and preparation. Catalysis with isobutanol selectivities as great as 70-80 wt\% were developed, but the overall alcoinol yield was only $11 \mathrm{lb} / \mathrm{ft}^{3} / \mathrm{hr}$. It was shown that catalyst performance is more dependent on the catalyst formulation than the catalyst preparation techniques.

Our immediate goal is to map the response surface of the crucial variables using fractional factorial experimental designs. Initial goals in our catalyst development program are to increase oxygenate selectivities to greater than $90 \%$ with methanol making up less than $70 \%$ of the liquid product, and increase rates to $20 \mathrm{lb} / \mathrm{ft}^{3} / \mathrm{hr}$ with conversions of greater than $20 \%$.

\subsubsection{Catalyst Synthesis Results}

A systematic search of the literature has been initiated to ensure that our literature base on conversion of syngas to higher alcohols is current. This activity will be continued throughout the project.

One area identified for initial investigation on the catalyst preparation front is the influence of precipitation $\mathrm{pH}$ on surface area, porosity, and thermal characteristics of the resulting precipitate. Potentiometric titrations during coprecipitation were conducted to aid in improving this catalyst preparation technique. Potentiometric titrations were carried out during coprecipitation from aqueous solutions containing zinc, chromium, and manganese nitrates. Titration curves of $\mathrm{pH}$ versus $\mathrm{CO}_{3}{ }^{2-/}$ total $\mathrm{M}^{\mathrm{n}+}$ have shown, for example, that if a coprecipitation is to be carried out at a constant $\mathrm{pH}$ of 9 , the rate of addition for the respective solutions should be such that the $\mathrm{CO}_{3} 2 \%$ total metal ratio is 1.7 . Further experiments are in progress in this area.

Variables in catalyst formulation and preparation will be examined by a fractional factorial designed experiment. The variables are based upon previous work at UCC on a catalyst system that yielded an isobutanol-rich product. A 16-run, 8-variable fractional factorial design has been set up to study the following variables: $\mathrm{Zn} /(\mathrm{Min}+\mathrm{Cr}), \mathrm{Mn} / \mathrm{Cr}$, calcination 
temperature, calcination time, levels of potassium and two other metals, and reduction temperature. Other variables such as precipitating $\mathrm{pH}$, drying temperature and time, and reduction time will be fixed at reasonable values. A $3 \times 3$ design is being carried out to determine suitable values for the precipitating $\mathrm{pH}$ and the range for calcination temperature and time.

\subsubsection{Catalyst Evaluation}

\subsubsection{Introduction}

A microreactor system has been revamped for use in screening heterogeneous catalysts in this project. Up to three fixed-bed reactor tubes may be controlled independently except for reaction temperature.

\subsubsection{Microreactor System for Screening Catalysts}

The Syngas Microreactor system is designed to screen heterogeneous catalysts for the conversion of synthesis gas to methanol and higher alcohols over a pressure range of 15$1500 \mathrm{psig}$, a temperature range of $25-400)^{\circ} \mathrm{C}$, and feed gas flow rate range of $50-1000 \mathrm{sccm}$. Up to three fixed-bed reactor tubes may be operated independently, except for reaction temperature. The unit consists of three sections: the feed section, the reaction section, and the analytical section (Figures 1, 2, and 3).

1. Feed Section. The feed section is designed to allow the operator to select any one of four premixed feed gases independently for any reaction tube. In addition, a nitrogen purge and pressure testing stream is available for selection. After stream selection, the feed gas for a reaction tube is passed through a molecular sieve/activated carbon trap to remove metal carbonyl contaminants. The purified gas then passes through a mass flow meter and into the reaction tube inlet.

2. Reaction Section. Each reaction tube is manufactured from $0.5^{\prime \prime}$ stainless steel and can hold up to ten milliliters of catalyst. The reaction tubes are heated using an air fluidized sandbath which is operated by a PID controller that senses the process temperature via a thermocouple positioned directly in the sandbath. The temperature of each reaction tube is also monitored using a thermocouple positioned axially inside the reaction tube. The reaction tube offgas passes into a spring-loaded back pressure regulator (used to elevate reaction pressure) equipped with a bypass valve.

3. Analytical Section. From the back pressure regulator, the product gas stream enters into the analytical section of the unit. The analytical section consists of automated, airactuated, gas sampling valves (GSVs) to provide on-line offgas sampling, followed by knock-out drums to collect liquid product for future analyses. 
Figure 1. Feed System of Syngas Microreactor System

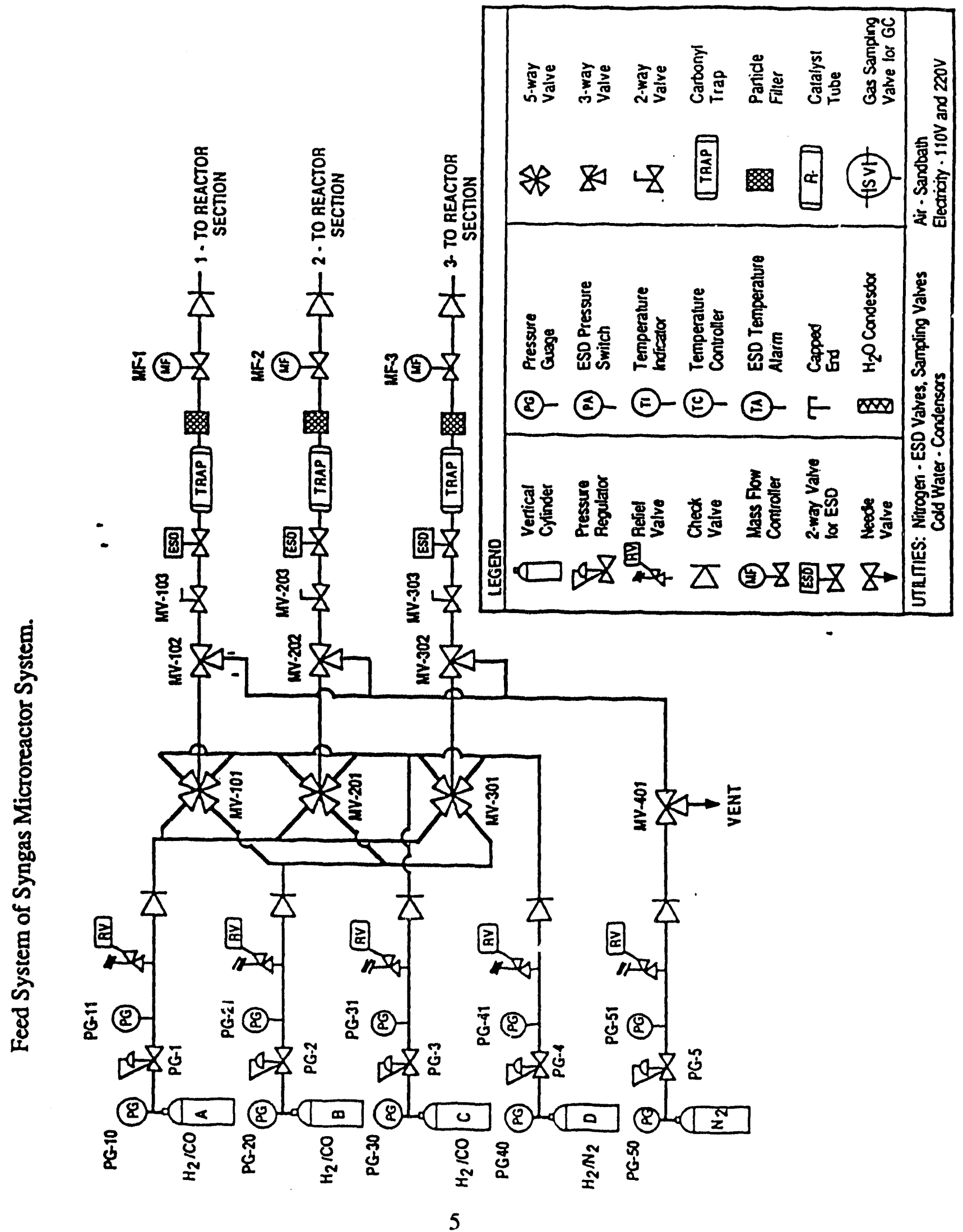


Figure 2. Reactor System of Syngas Microreactor System

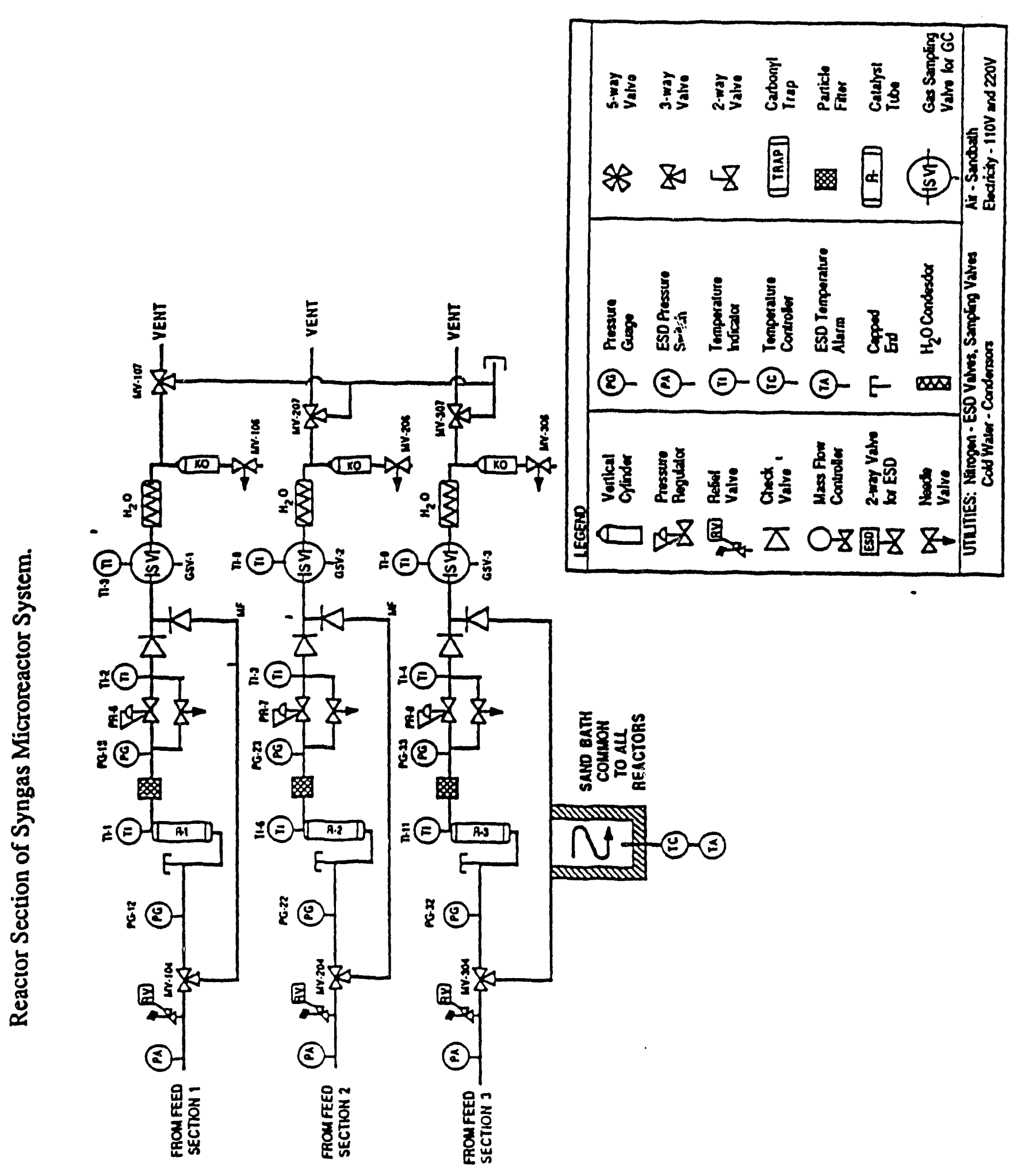


Figure 3. Analytical System of Syngas Microreactor System
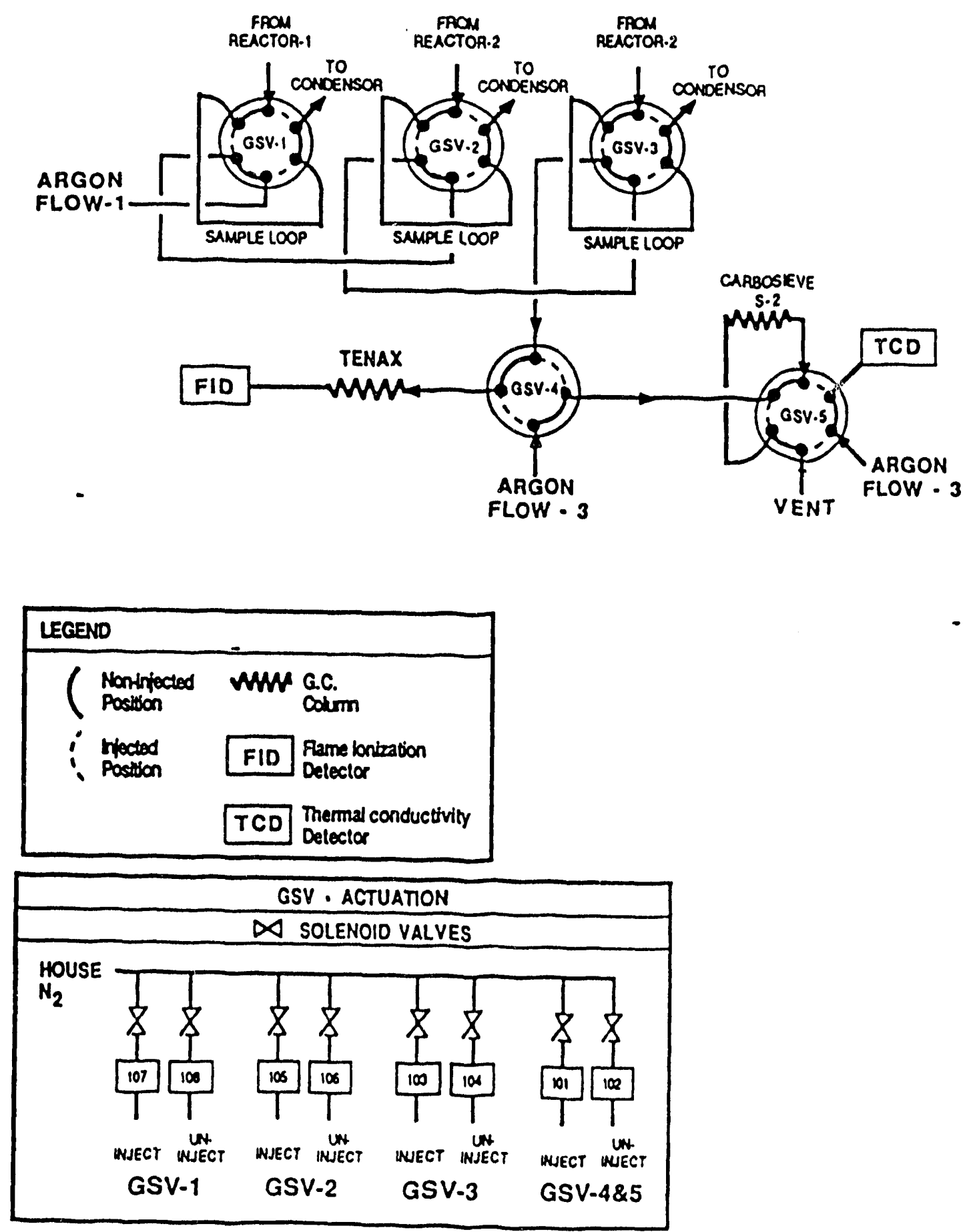


\subsubsection{Product Analysis}

Each reaction line in the microreactor system passes through an independent six port, two position, gas sampling valve (GSV-1, GSV-2, or GSV-3). These valves alternatingly provide $0.6 \mathrm{ml}$ reactor offgas samples to a Varian 3700 gas chromatograph. All valves are pneumatically actuated using a collection of solenoid valves controlled via the UCC computer automated laboratory system (CALS).

Once a sample enters the argon carrier gas stream (see Figure 3), it is transported outside of the fume hood to a four-port two-position air-actuated switching valve (GSV-4) located at the GC. This valve directs the sample to either the organics separation (Tenax column) and detection leg of the analysis scheme or to the inorganics separation (Carbosieve S-2 column) and detection section. While GSV-4 is directing samples to the Tenax column, GSV-5, a sixport two-position gas sampling valve back-flushes the Carbosieve column of accumulated organic compounds from previous analyses. GSV-4 and GSV-5 are bridged so that they actuate at the saine moment. The analytical section is designed to have GSV-1, 2 or 3 send two samples to the GC two minutes apart. The first sample goes to the organics analysis section. On the second sampling, GSV-4 and 5 are actuated to divert the second gas sample to the inorganics analysis section.

\subsubsection{Gas Chromatographic Analyses}

A single Varian 3700 gas chromatograph equipped with two detectors is used for offgas analysis. Inorganics $\left(\mathrm{H}_{2}, \mathrm{~N}_{2}, \mathrm{CO}\right.$, and $\left.\mathrm{CO}_{2}\right)$ are separated on a 10'x1/8" 80/100 mesh Carbosieve S-2 column purchased from Supelco and detected by thermal conductivity. All organic products are resolved on a $12^{\prime} \times 1 / 8^{\prime \prime} 80 / 100$ mesh Tenax column obtained from Alltech and detected using flame ionization. The Tenax column has been calibrated on an absolute weight basis using quantified mixtures of $\mathrm{C}_{1}-\mathrm{C}_{6}$ normal hydrocarbons, normal and branched alcohols, and normal aldehydes. Argon is used as the carrier gas for both columns. The following parameters are used for the GC oven and the two columns and detectors:

Temperature ramp: $50^{\circ} \mathrm{C}$ to $225^{\circ} \mathrm{C}$ at $8^{\circ} \mathrm{C} / \mathrm{min}$ with a 10 minute hold at the upper temperature

Column

Tenax

Carbosieve (sample)

Carbosieve (reference)
Flow Rate

$20 \mathrm{sccm}$

$20 \mathrm{sccm}$

$20 \mathrm{sccm}$
Injector Temp.

$220^{\circ} \mathrm{C}$

$170^{\circ} \mathrm{C}$

$170^{\circ} \mathrm{C}$ 


\begin{tabular}{|c|c|c|c|c|}
\hline Detector & Temperature & $\underline{\text { Sensitivity }}$ & Attenuation & Qther \\
\hline TCD & $200^{\circ} \mathrm{C}$ & $5.0 \mathrm{mV}$ & $4 X$ & Filament Temp. $=350^{\circ} \mathrm{C}$ \\
\hline FID & $250^{\circ} \mathrm{C}$ & $10^{-10}$ & $8 \mathrm{X}$ & $\begin{array}{l}\mathrm{H}_{2} \text { flow }=30 \mathrm{sccm} \\
\text { Air flow }=300 \mathrm{sccm}\end{array}$ \\
\hline
\end{tabular}

\subsubsection{Data Analysis}

Upon collecting the dual channel GC chromatogram for a reactor tube, CALS determines the identity and area of all components eluted and passes the results to a VAX mainframe computer. The cperator accesses the VAX using a terminal in the laboratory and enters a FORTRAN prograni that has been written to provide further data analysis. The operator inputs the feed and offgas flow rates, reaction conditions, and catalyst reference numbers. The program calculates the catalyst activity and selectivity as well as performing carbon, hydrogen, and oxygen mass balances. In general, the oxygen and hydroger: mass balance is not $100 \%$ because the analytica! system is unable to quantitate the $\mathrm{H}_{2} \mathrm{O}$ concentration in the offgas. A summary of the results may then be printed in a convenient format.

\subsubsection{Task 1 Conclusions}

Literature and laboratory work have begun on the preparation of catalysts for conversion of syngas to fuel alcohols. The initial work will be on the isobutanol catalyst system. Future work will include copper-based catalysts, dealing with different aspects of the support, the impregnation of the metals, and choice of promoters.

A microreactor system has been prepared for use in screening heterogeneous catalysts in this project. It will allow the independent control of three fixed-bed reactor tubes except for reaction temperature. Appropriate temperature, pressure, and flow ranges are attainable with this system.

\subsection{Task 2 - Engineering Studies}

\subsubsection{Reaction Engineering}

4.2.1.1. Introduction

Task 2 involves reaction engineering studies to examine the best catalysts identified in Task 1. New catalysts will be developed and screened in powder form in Task 1. Task 2 work will develop scaleup and process strategies to make use of the best catalysts found in Task 1 and will develop kinetic models to describe their performance.

Since powders cannot be used commercially in a gas-phase reactor (the type which will be used for initial screening studies), we will evaluate different strategies for utilizing new catalysts on an industrial scale. This may include pelletizing or tableting the powdered catalysts for use in gas-phase reactors, using powdered catalysts in a gas-liquid-solid slurry 
reactor, or using solid pellets or powder in other types of three-phase reactors, such as trickle bed reactors.

The facilities available for Task 2 work will be briefly described here.

\subsubsection{Reaction Engineering Facilities}

Two Reaction Engineering laboratories are available for preparing and testing catalysts: a catalyst preparation facility and a catalyst testing facility. The catalyst preparation facility has general catalyst scaleup and preparation equipment and facilities for small scale, liquid phase catalyst testing. This facility will be used primarily for preparing extrudates or pellets from powdered catalysts. The major catalyst preparation equipment in this lab include:

(1) Drying oven $\left(350^{\circ} \mathrm{C}\right.$ maximum)

(2) Calcining oven $\left(1250^{\circ} \mathrm{C}\right.$ maximum, $\mathrm{N}_{2}$ purge available)

(3) Rotavap (vacuum, various purges, and heating to $120^{\circ} \mathrm{C}$ available) and various glassware for impregnating, mixing, and binding powders

(4) Small lab-scale extruder for forming pellets

In the Reaction Engineering Catalyst Testing and Development Laboratory, four reaction systems are available for testing catalysts and obtaining kinetic data:

(1) Tubular microreactors for small-scale gas-solid reactions

(2) Berty autoclave for gas-solid kinetic studies

(3) Slurry autoclave for three-phase reactions

(4) SpectraTech in situ FTIR reaction system for online analysis of products and intermediates.

The microreactor system will be used for testing pelleted (large scale) catalysts in gas-phase systems, the Berty reactor for detailed kinetic studies, the slurry reactor to evaluate slurry (three phase) process schemes using a powdered catalyst, and the SpectraTech FTIR reaction system to obtain kinetic data and information about intermediate products.

All systems can be used at high pressures and temperatures. The inaximum operating conditions and volumes for each are listed below:

(1) Microreactors: $400^{\circ} \mathrm{C}, 900 \mathrm{psig}, 30 \mathrm{ml}$

(2) Berty reactor: $375^{\circ} \mathrm{C}, 1000 \mathrm{psig}, 300 \mathrm{ml}$

(3) Slurry: $290^{\circ} \mathrm{C}, 1500 \mathrm{psig}, 2$ liter

(4) SpectraTech: $200^{\circ} \mathrm{C}, 1100 \mathrm{psig}, 300 \mathrm{ml}$. 
All systems are controlled (temperatures, pressures, feed rates) by a Texas Microsystems computer running the FIX software, providing 24 hour unsupervised operation. This system also acquires and stores data. The microreactors and Berty and slurry reactors are connected to an online GC for unattended analysis of products. Analyses for the SpectraTech are done using FTIR through an internal reflectance crystal located in the base of the autoclave.

Finally, another Reaction Engineering facility inay be available during the course of this study. It contains a large-scale multiphase reactor and control equipment (Texas Microsystems computer with FIX software) for conducting reaction and flow dynamics studies of gas-solid-liquid reactions, such as in a trickle bed reactor.

\subsubsection{Mathematical Process Development}

\subsubsection{Introduction}

Mathematical process development can be broken down into synthesis and simulation. Process synthesis determines which unit operatioris are needed and in what order. Once the structure is defined by the synthesis step, the process is simulated to determine the heat and material balance. Software tools available for process synthesis and simulation are used to:

- Optimize operating conditions

- Study process alternatives

- Evaluate potential of new technology developed in the laboratory

- Develop process economics

- Provide direction for further lab experiments

\subsubsection{Software Programs to be Used}

Process Synthesis Software. Two software programs are available for process synthesis. The first is the Mayflower Program for separation system synthesis. It is based on the generation of residue curve maps which define a feasible separation region. The second process synthesis tool is Advent which is used for heat exchanger network synthesis. The program is based on pinch technology and defines the minimum utility requirements of a process. It will also determine the optimum tradeoff between energy and capital cost.

Simulation Software. Aspen Plus software is used to simulate a process flowsheet and determine a heat and material balance. It can simulate a variety of unit operations and provides sizing information needed to determine equipment cost. The program can also determine the sensitivity of operating variables on the process.

\subsubsection{Task 2 Conclusions}

Various process schemes will be evaluated and kinetic data will be obtained using the best candidate catalysts found in Task 1 to examine their commercial potential in large-scale processes. Powdered catalysts will be converted into large-scale materials (pellets, extrudates) and tested in gas-solid reactors, or examined as powders in reaction systems that 
can handle these materials (slurry reactor, multiphase reactor). Bench-scale equipment is available for these studies.

Process conceptualization and the determination of optimum operating conditions are achieved through process synthesis. Software is available to aid in the task. Process synthesis is also instrumental in defining directions for further laboratory experiments. 

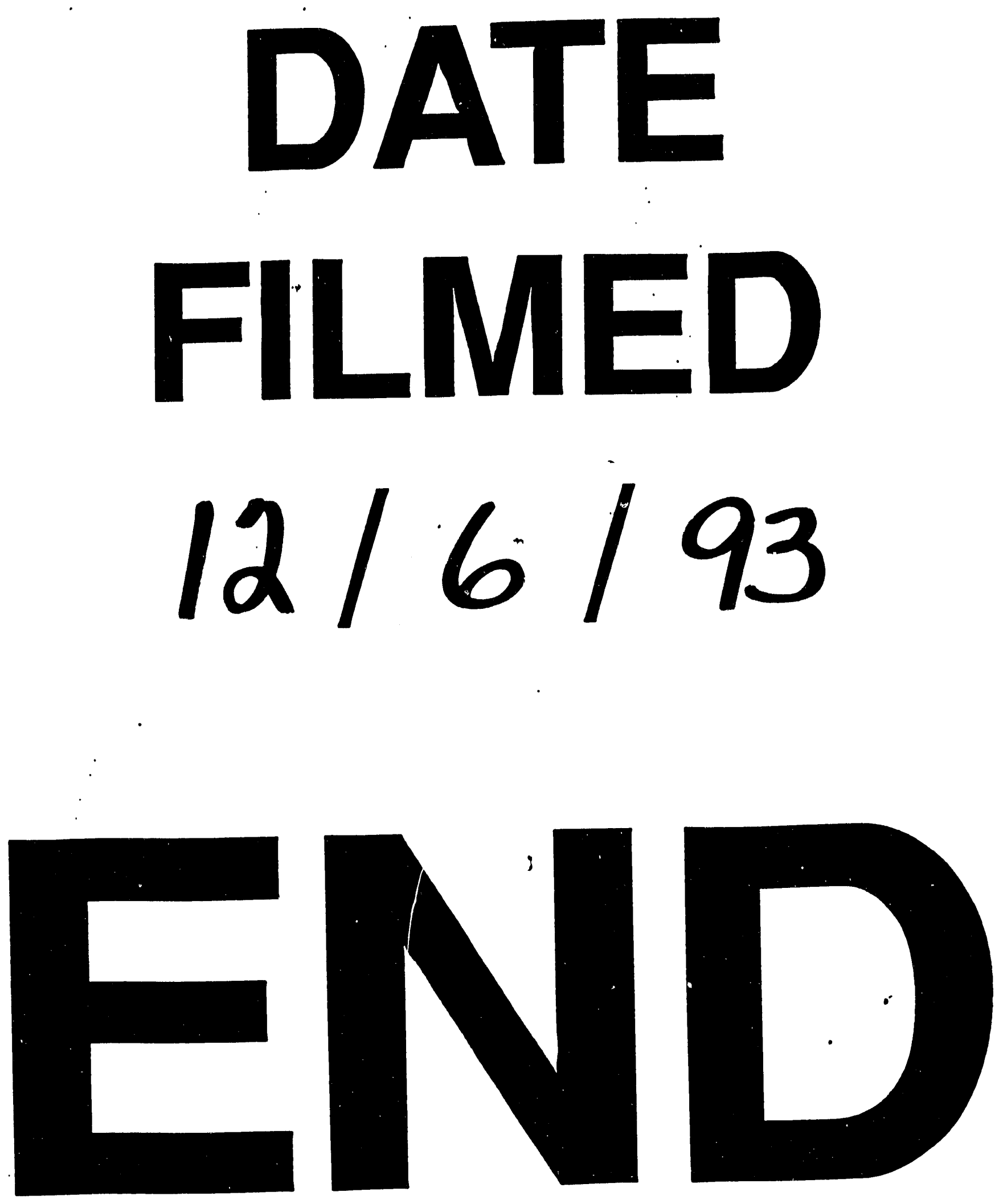
